SINGLE PASS COLLIDER MEMO
AUTHOR: J. J. Murray, T. Fieguth and S. Kheifets
DATE:
July 23, 1986
TITLE:

\section{Dispersive Effects of Transverse Displacements of SLC Arc Magnets}

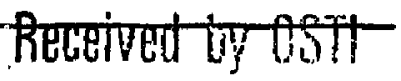

\title{
Summary
}

JUL 31 1996

The SLC Arc magnets are aubject to random displacements and field errors resulting in unpredictable transverse displacement of the centrai trajectory from that of the design. The chosen method of correcting this perturbed trijectory in the SLC Arcs utilizes mechanical moverrent of the combined functior magnets which compose the Arc transport lines. Here we present the resuits c: a recent investigation substantiating the earlier results which led to the adoption of this method.

\section{Introduction}

During the design phase of the SIC Arcs various correction methods were suggested and studied. Two of them were considered as most promising:

- use of Backleg Windings (BLW) and

- transverse translision of the combined function magnets, i. e. use of Magnet Movers (MM).

In the course of these studies one of the authors (JJM) first observed that trajectory correction by MM introduced significantly less anomalous dispersion than that introduced by BLW. This important observation was subsequently corroborated by others working on Arc Beam Dynamicsl using both analytic methods and computer simulations. Most of these efforts are documented in minutes of different meetings only and are not readily available.

However, the decision to use MM was based upon that easlier collaborative effort. What is reported here are the results of a recent review of the dispersive effec's of the MM including some original approaches not previously used.

The FODO cells of the SLC Arcs are put together to form second-order achromats. ${ }^{2}$ Jach cell is composed of two combined function magnets each having superimposed dipole, quadrupole and sextupole fields. The strength of the dipole field is the same for both magnets. The field gradients in both magnets are nea-ly 
the same but the signs are opposite. The sextupole components are different both in the signs and in the strengths.

In this lattice the dispersion function, defining the deviation of the trajectory for an off-momentum particle with momentum $p$ from that for the on-momentum particle with momentum $p_{o}$, has been matched in the ae: re that the dispervion function and its derivative with respect to the path leng are both periodic with a period equal to the cell length for an unperturbed (..e. no misalignment or field errors) syatem. This matched dispersion function and its derivative are called the $\eta$ and $\eta$ ' functions.

This statement must be qualified somewhat by pointing out that deviation from this perfectly "matched" condition are created by necessity of rolling some achromats as a whole to allow vertical deflections of the Arcs for following the site terrain. The effect of including these roll into the design has been studied but will not be discussed here.

Dipole field errors and transverse displacements of multip le fields will in general produce dispergive angular "kicks" ( momentum deper. - ?nt changes of direction) in a particle beam trajectory. The kicks are linear wi. : displacement of quadrupoles and quadratic with displacement of sextupoles. The momentum dependence of a kick can be charecterized by a change $\Delta \eta^{\prime}$ of the slope of the matched dispersion function. This change will propagate through the downctream syotom an anamalies of the functiond $\eta$ and $\eta^{\prime}$.

\section{Non-Dispersive Condition}

Consider a differential slice of an Are magnet with length ds. The tranoverse field components can be expressed as follows,

$$
\begin{gathered}
B_{y}=B_{0}+B_{0}^{\prime} x+\frac{1}{2} B_{0}^{\prime \prime}\left(x^{2}-y^{2}\right), \\
B_{x}=B_{0}^{\prime} y+B_{0}^{\prime \prime} x y
\end{gathered}
$$

where the coefficients $B_{o}, B_{o}^{\prime}$ and $B_{o}^{\prime \prime}$ represent the dipole, quadrupole and sextupole strengths of the combined function magnet, respectively.

Suppose at this point that there is no dispersion in the $y$ plane and that the horizontal dispersion is equal to 7 . Suppose furtiser that the elemental magnet is displaced from the reference axis by $\Delta x$ and $\Delta y$. Then, with $p=p_{0}(1+\delta)$,

$$
x=n \delta+\Delta x
$$




$$
\boldsymbol{y}=\Delta \boldsymbol{y}
$$

With these values inserted in Eqs. $(1,2)$, the angular "zicle" $d \theta$ in horizontal and $d \phi$ in vertical planes of a ray passing through the elemental magnet may be written as follows,

$$
\begin{gathered}
d \theta=\frac{d s}{p_{0}(1+\delta)}\left[\left(B_{0}+B_{0}^{\prime} \eta \delta+\frac{1}{2} B_{0}^{\prime \prime} \eta^{2} \delta^{2}\right)+\left(B_{0}^{\prime}+B_{0}^{\prime \prime} \eta \delta\right) \Delta x+\frac{1}{2} B_{0}^{\prime \prime}\left(\Delta x^{2}-\Delta y^{2}\right)\right] \\
d \phi=\frac{d o}{p_{0}(1+\delta)}\left[\left(B_{0}^{\prime}+B_{0}^{\prime \prime} \eta \delta\right) \Delta y+B_{0}^{\prime \prime} \Delta x \Delta y\right] .
\end{gathered}
$$

The first term in $d \theta$ is simply the deflection produced by the unperturbed magnet and is of no interest here.

Let us cunsider the linear approximation in $\Delta z$ and $\Delta y$ and consequently neglect all quadratic terms in these quantities. Then we have

$$
\begin{aligned}
& d \theta \approx \frac{d s}{p_{0}(1+\delta)}\left(B_{0}^{\prime}+B_{0}^{\prime \prime} \eta \delta\right) \Delta x, \\
& d \phi \approx \frac{d s}{p_{0}(1+\delta)}\left(B_{0}^{\prime}+B_{0}^{\prime \prime} \eta \delta\right) \Delta y .
\end{aligned}
$$

Clearly, both $d \theta$ and $d \phi$ will be approximately independent of momentum if the following condition is satisfied,

$$
B_{0}^{\prime \prime} \eta \approx B_{0}^{\prime}
$$

This condition cannot be satisfied at all points in an arc magnet since within a magnet $\eta$ varies while $B_{o}^{\prime}$ and $B_{o}^{\prime \prime}$ do not. To make a gyatem non-dispersive we should use $\bar{\eta}, i$, en an $\eta$ veraged over the magnet segment actually being roved:

$$
B_{0}^{\prime \prime} \bar{\eta}=B_{0}^{\prime}
$$

Eq. (10) is the non-dispersive condition. 
Neglecting the short drift between magnets, the matched $\eta$-function in the FODO lattice can be expressed as follows,

$$
\begin{aligned}
& \eta_{F}=\eta_{\text {max }}+A_{F}\left(\cos \frac{s}{\lambda}-1\right), \\
& \eta_{D}=\eta_{\min }+A_{D}\left(\cosh \frac{s}{\lambda}-1\right),
\end{aligned}
$$

where $s$ is distance from the center of a magnet measured along the reference axis and

$$
\lambda=\left\{\left.\frac{p_{0}}{B_{0}^{t}}\right|^{\frac{1}{2}}\right.
$$

$A_{F}$ and $A_{D}$ are determined by the requirement that $\eta_{F}$ and $\eta_{D}$ join smoothly at the junction between magnets where $s=L / 4$ and $L$ is the length of a cell. The subscripts $F(D)$ refer to fccussing (defocussing) magnets.

In MURTLE simulation, translations, both random and deliberate, are applied to half magnets. In that case the average $\eta$ 's for $s$ between 0 and $L / 4$ are given by

$$
\begin{aligned}
& \overline{\eta_{F}}=\eta_{\text {max }}+A_{F}\left(\frac{\sin \alpha}{\alpha}-1\right), \\
& \overline{\eta_{D}}=\eta_{\min }+A_{D}\left(\frac{\sinh \alpha}{\alpha}-1\right),
\end{aligned}
$$

where $\alpha=L / 4 \lambda$.

The critical quantity, $B_{0}^{\prime \prime} \bar{\eta} / B_{0}^{\prime}$, has been evaluated in four cases, namely, for $F$ and $D$ versions of SLC ArC magnets (108' phase shift per cell) and for $F$ and $D$ versions of the magnets of an achromat sinilar to the SLC achromat but with $135^{\circ}$ phase shift per cell. 
Key parameters at $\mathbf{5 0} \mathrm{GeV}$ in these cases are listed in Table 1.

Table 1

\begin{tabular}{|c|c|c|c|c|c|}
\hline Magnet & $B_{0}^{\prime}$ & $B_{0}^{\prime \prime}$ & $\eta_{\text {maz, min }}$ & $\bar{n}$ & $B_{0}^{\prime \prime} \overline{7} / B_{0}^{\prime}$ \\
\hline & $\mathrm{kg} / \mathrm{cm}$ & $\mathrm{kg} / \mathrm{cm}^{2}$ & $\mathrm{~cm}$ & $\mathrm{~cm}$ & \\
\hline \multicolumn{6}{|c|}{ phase/cell $108^{\circ}$} \\
\hline FFOC108 & 7.014 & 1.621 & 4.743 & 4.296 & 0.993 \\
\hline Droc108 & -7.014 & -2.710 & 2.273 & 2.651 & 1.024 \\
\hline \multicolumn{6}{|c|}{ phase/cell $135^{\circ}$} \\
\hline FFOC135 & 8.034 & 2.367 & $\mathbf{3 . 7 5 7}$ & 3.363 & 0.981 \\
\hline DFOC135 & $-8,034$ & -4.310 & 1.600 & 1.926 & 1.094 \\
\hline
\end{tabular}

As demonstrated in this Table, Eq. (10) is satisfled within a few percent for the approximation used to evaluate $\bar{\eta}$ for these two and probably all second-order achromats.

\section{Simulations Results}

We have shown by Eqs. $(7,8$ and 10$)$ that the direction of the beam can be changed non-dispersively by magnet movement in the linear approximation in its displacement. The suppression of the linear effects and the onset of non-linear behavior has been studied for a single Arc achromat using the code MURTIE.

The results of these calculations are illustrated in Figures. 1 through 6 . The model used in these simulations is that of a single Arc achromat starting from the symmetry point of a focusing magnet. Each of the 20 magnets has been divided into two equal halves. These half-magnets are numbered sequentially from 1 to 40. Those halves designated as $4,8,12, \ldots, 40$ then represent horizontal magnet movers, whereas numbers $2,6,10, \ldots, 38$ correspond to vertical movers. Particular movers $4,6,8$ and 40 were chosen for these illustrations and in each case the effect of a horizontal or vertical translation was measured by calculating the change of $\eta$ and $\eta^{\prime}$ as an expansion about the perturbed orbit at the end of the achromat. Two series of calculations were performed with and without the sextupole field component in the half-magnet that, was moved to generate the non-dispersive and dispersive kicks, respectively. In general, with the exception of the last halfmagnet No. 40, these kicks initiate a free betatron oscillation in the downstream magnets. Thus the changes of $\eta$ and $\eta^{\prime}$ are the cummulative result of the initial and subsequent kicks due to orbit displacement in these magnets. Whether the effect is most noticeable as a change in $\eta$ or $\eta^{\prime}$ as measured at the end of the achromat depends upon the relative phase shift of the moved magnet. 
In Fig. 1 the changes in $\eta_{x}$ and $\eta_{x}^{\prime}$ produced by horizontal displacement of the mover No. 4 are shown. The phase shift for this mover is close to the multiple of $\pi / 2$. As expected it is $\eta$ which demonstrates the linear effect when the sextupole term of this mover is turned off. Fig. 2 illustrates the effect of the horizontel displacement of the mover No. 8. For this mover the phase shift is approximately a multiple of $\pi$ so the linear effect shows up predominantly in $\Delta \eta^{\prime}$.

In Figs. 3, 4 and 5 the effects of the vertical magnet mover No. 6 are shown for both vertical and horizontal displacements. Fig, 3 illustrates the broad range of vertical movement without large second-order effects on $\eta_{y}$ and $\eta_{y}^{\prime}$ as can be seen from eq. (6) when $\Delta x$ is put to zero. On the other hand Fig. 4 shows the aecond-order effects on $\eta_{m}$ and $\eta_{E}^{\prime}$ with vertical displacement as predicted by eq. (b). Note especially, that the behavior is similar for the aextupole either turned on or off. This is because the perturbed orbit has vertical offeet in all downstream magnets.

Fig. 5 illustrates the effect of a horizontal offset in half-magnet No. 6 where $\beta_{x}$ and $\eta_{a}$ are at their respective minima. The resultant downstream trajectory is $\approx 3$ times smaller in amplitude than that caused by mover No. 8 in Fig. 2, which is at the maximum of $\beta_{x}$. The effect on $\eta_{y}$ and $\eta_{y}^{\prime}$ of moving this half-magnet No. 6 horizontally is negligible and is not illustrated.

Fig. 6 is plotted for the last magnet mover No. 40 so there are no contributions to $\Delta \eta$ and $\Delta \eta$ ' stemming from the perturbed orbit in the downstream magnets. This figure most clearly illustrates that the non-dispersive feature is the consequence of the non-dispersive condition (10). Such a combined function magnet can be used as a non-dispersive orbit corrector in any arbitrary transport system if positioned at a point of non-zero dispersion.

In view of nonlinearities exhibited by the simple system modeled above, one might speculate that in a complete Arc with all magnets subject to random translations their cumulative effect might contribute significantly to the onset of uncorrectable nonlinear effects observed to occur with rms displacements in the $\mathbf{2 0 0}$ to $\mathbf{3 0 0}$ micron range.

\section{Conclusions}

Based on these observations we conclude that the magnets in the SLC achromats satisfy the non-dispersive condition (10) with respect to translations and that magnets in any $\eta$ matched, second-order achromat probably do 80 also. But it is clear that for any $\eta$ matched, periodic lattice not necessarily composed of second-order achromats, values of $B_{0}^{\prime \prime}$ can always be found such that the rondispersive conditions for translation are satisfied in both $F$ and $D$ magnets.

When the non-dispersive condition (10) is satisfied the linear dispersive effects in magnet displacements are suppressed but the higher-order terms are still present and distort the dispersion of the system. It is these and other nonlinear effects that have limited the tolerance for displacements in the SLC Arcs. 


\section{REFERENCES}

1. Private communications of the Beam Dynamics Task Force (BDTF). At the time of the decision to use MM the group consisted of $K$. Brown, A. Chao, J. Jaeger, S. Kheifets, J. Murray, R. Servrancke and $\boldsymbol{H}$. Shoaee.

2. K. L. Brown, “A Second-Order Magnetic Optical Achromat", SLAC-PUB2257, February 1979 

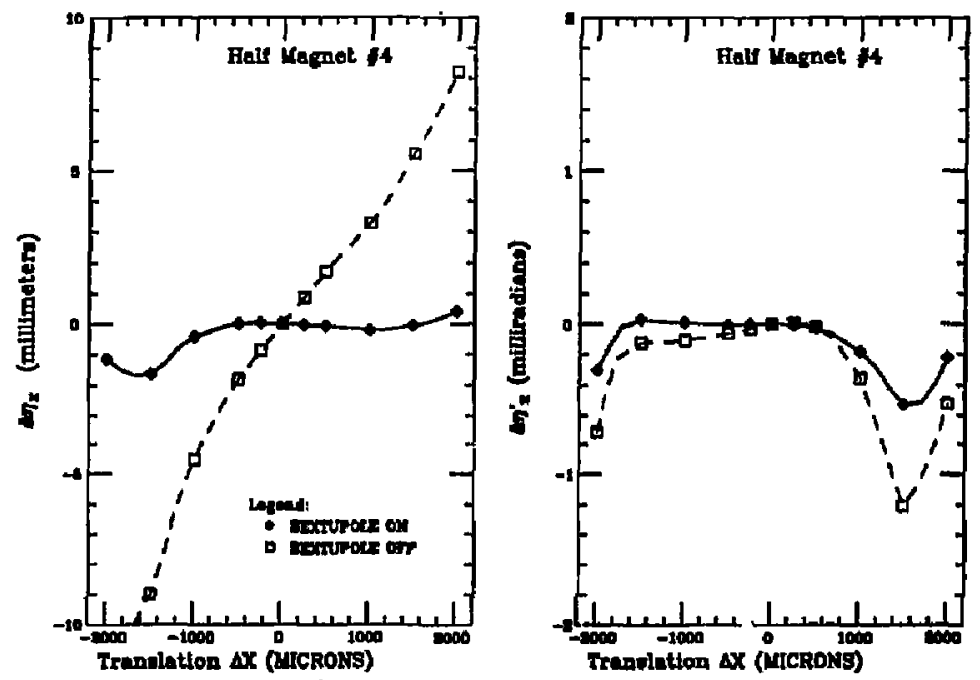

Fig. 1. Effect on $\eta_{2}, \eta_{z}^{\prime}$ for horiz. displacement of half magnet 4 (F).
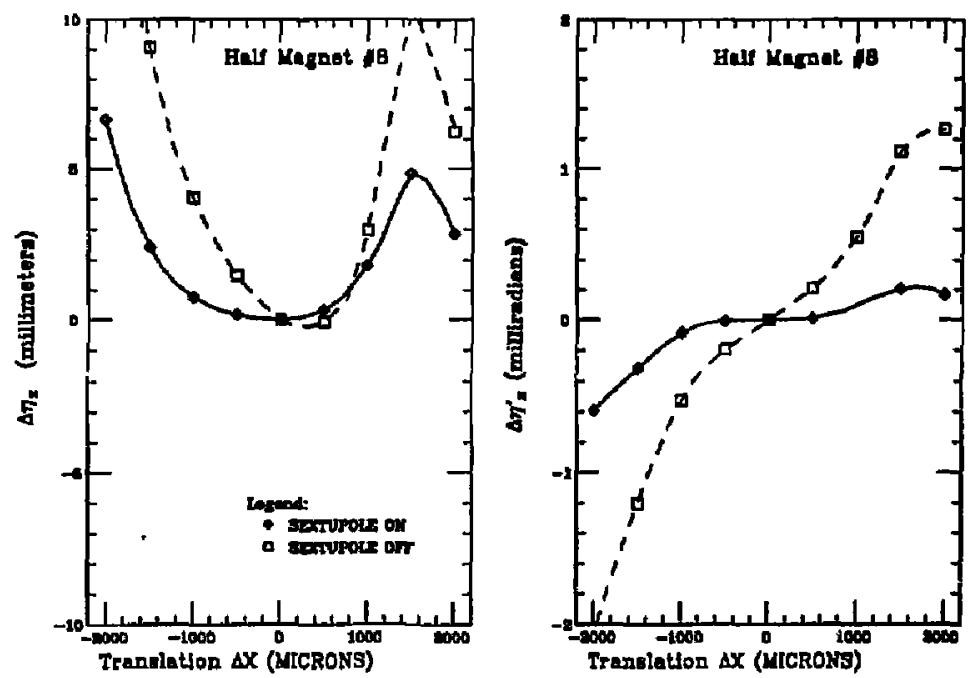

Fig. 2. Effect on $\eta_{x}, \eta_{x}^{\prime}$ for horiz. displacement of half magnet 8 (F). 

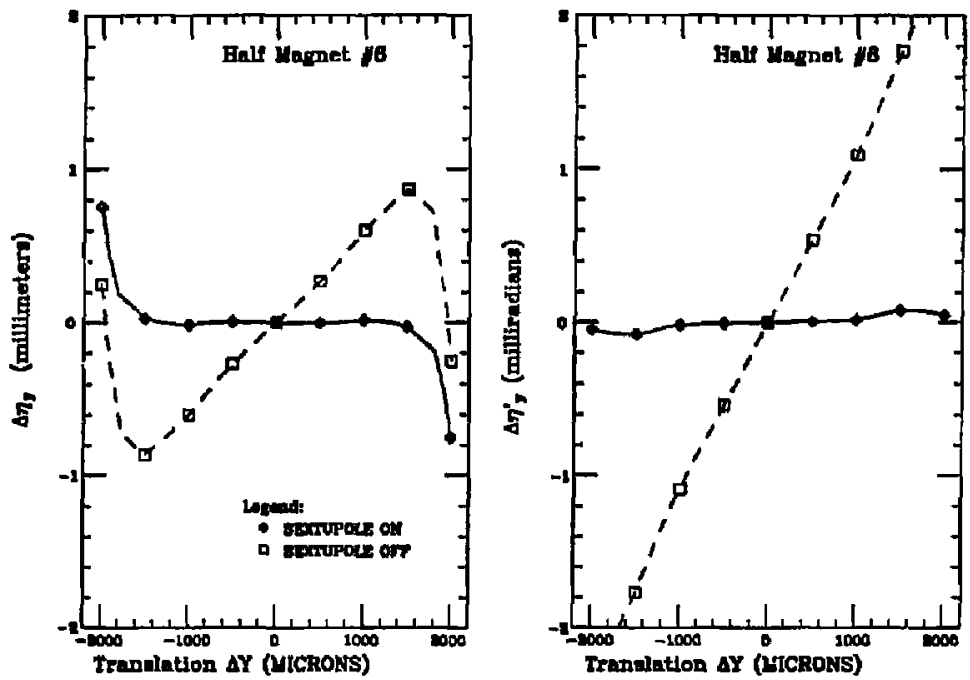

Fig. 3. Effect on $\eta_{y}, \eta_{y}^{\prime}$ for vert. displacement of half magnet 6 (D).
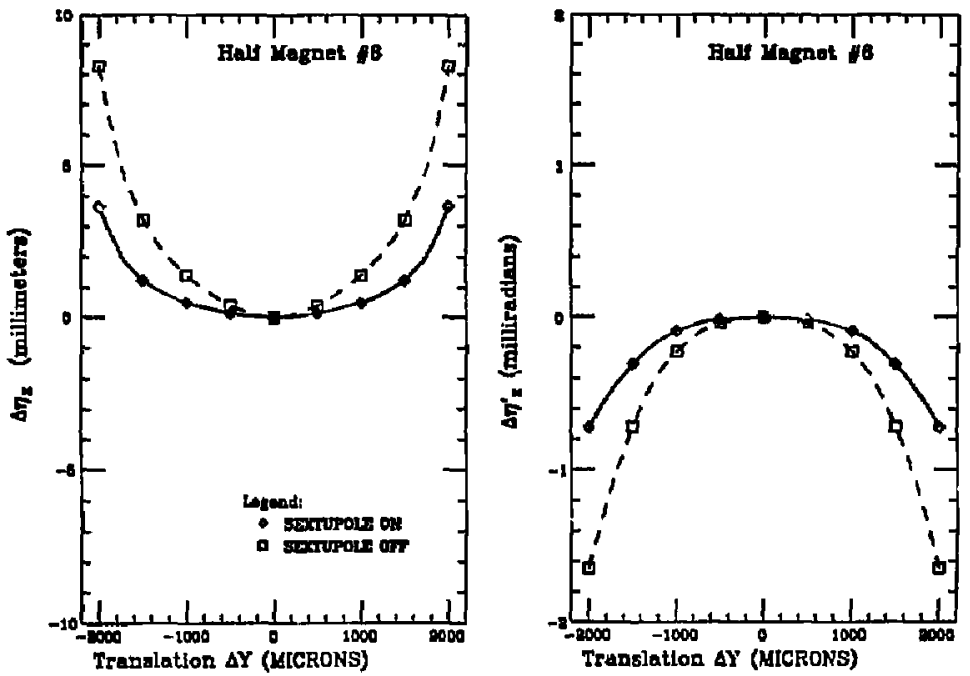

Fig. 4. Effect on $\eta_{x}, \eta_{x}^{\prime}$ for vert. displacement of half magnet 6 (D). 

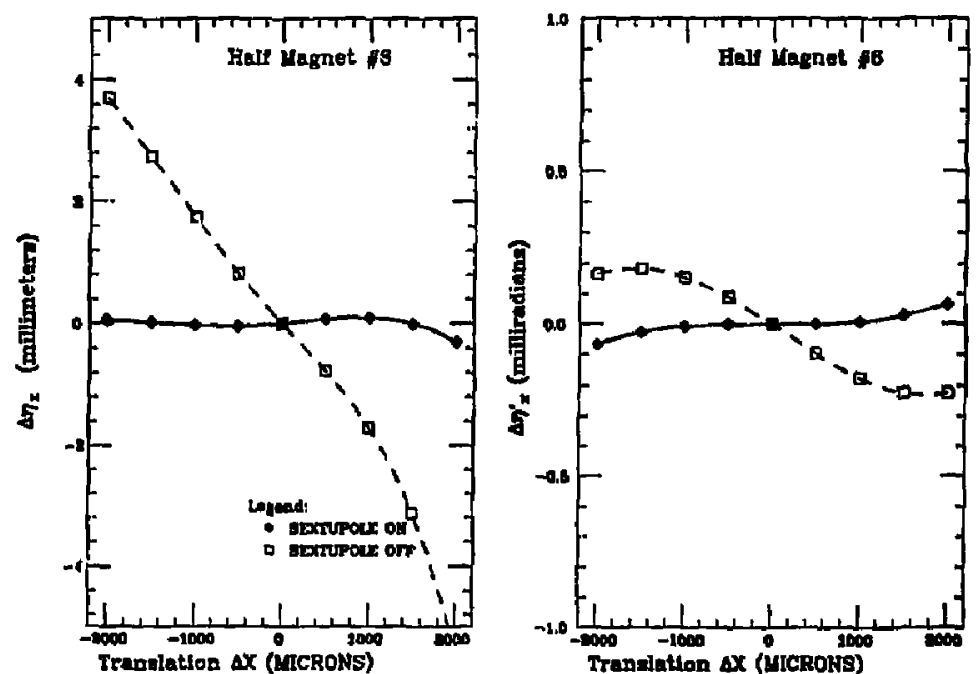

Fig. 5. Effect on $\eta_{x}, \eta_{x}^{\prime}$ for horiz. displacement of half magnet 6 (D).
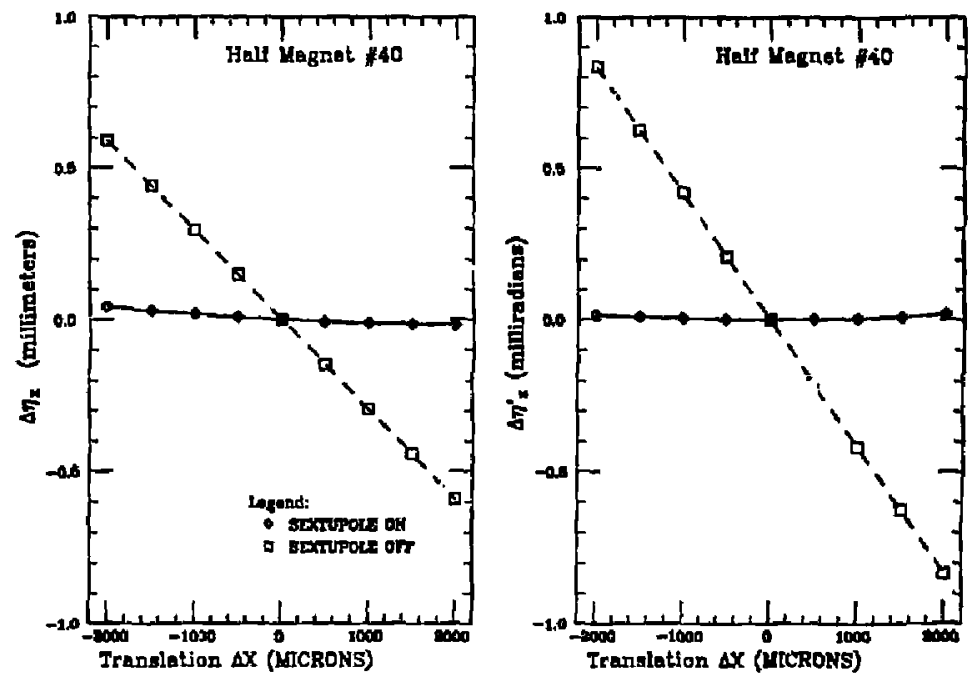

Fig. 6. Effect on $\eta_{x}, \eta_{x}^{\prime}$ for horiz. displacement of half magnet 40 (F). 


\section{DISULAIMER}

This report was prepared as an account of work sponsored by an agency of the United States Government. Neither the United States Government nor any agency thereof, nor any of their employees, makes any warranty, express or implied, or assumes any legal liability or responsibility for the accuracy, completencss, or usefulness of any information, apparatus, product, or process disclosed, or represents that its use would not infringe privately owned rights. Reference herein to any specific commercial product, process, or service by trade name, trademark, manufac:urer, or otherwise does not necessarily constitute or imply its endorsement, recommendation, or favoring by the United States Government or any agency thereof. The views and opinions of authors expressed herein do not necessarily state or reflect those of the United States Government or any agency thercof. 